\title{
Rise time in shock consolidation of materials
}

\author{
W. Tong \\ Department of Engineering Mechanics, University of Nebraska-Lincoln, Lincoln, Nebraska 68588-0347 \\ G. Ravichandran \\ Graduate Aeronautical Laboratories, California Institute of Technology, Pasadena, California 91125
}

(Received 20 May 1994; accepted for publication 22 September 1994)

The rise time of a strong shock wave propagating through a porous material is estimated by analyzing the finite deformation of an elastic/viscoplastic spherical shell under impulsive pressure loading. The analysis examines explicitly the effects of dynamic loading rate and initial temperature and explains the relatively large shock rise times observed in porous materials. Results of the analysis provide a consistent and realistic interpretation of available experimental data. (C) 1994 American Institute of Physics.

Metallic and ceramic powders can be consolidated by the passage of a strong shock wave through an initially porous media. ${ }^{1-4}$ Earlier investigations of shock wave phenomena in porous materials have focused primarily on the shock Hugoniot relationships and the minimum pressure required to produce a fully dense compact. ${ }^{5,6}$ In addition, the processes associated with shock consolidation have been investigated experimentally and theoretically. ${ }^{7-9}$ A finite rise time of shock waves in porous materials has been observed ${ }^{7,8}$ which was independent of pressure at high shock pressures. ${ }^{8}$ The collapse of a spherical shell under an external impulsive pressure loading has been studied by Carroll and Holt ${ }^{9}$ to model dynamic compaction of porous materials. The deformation of the matrix material was assumed to be rate independent, perfectly plastic. The finite rise time of the shock wave propagating through a porous material was found to be related to the time of pore collapse and it was shown that pore collapse is retarded by inertial effect.

The dynamic consolidation process involves high strain rates in the range $10^{5}$ to $10^{7} \mathrm{~s}^{-1}$. Experimental evidence indicates that flow strength of most metals is strongly rate dependent at these strain rates. ${ }^{10,11}$ Tong and Ravichandran ${ }^{12}$ have recently reexamined the dynamic pore collapse problem by considering the finite elastic/viscoplastic deformation of a spherical shell. Their results indicated that strain rate, strain hardening, thermal softening, dynamic loading rate, pore size, and initial relative density play a significant role in pore collapse. In particular, a viscoplastic material model including strain-rate sensitivity, strain hardening, and thermal softening is assumed to be of the form ${ }^{10-12}$

$$
\tau=\tau_{0}\left(\frac{\dot{\gamma}}{\dot{\gamma}_{0}}\right)^{m}\left(\frac{\gamma}{\gamma_{0}}\right)^{n}\left(\frac{\theta}{\theta_{0}}\right)^{\nu}
$$

where $\tau, \dot{\gamma}, \gamma$, and $\theta$ are shear flow stress, shear strain rate, plastic shear strain, and temperature, respectively, with the corresponding reference values indicated by the subscript "0." Parameters, $m, n$, and $\nu$ describe the rate sensitivity, strain hardening, and thermal softening of the material. Table I lists the viscoplastic parameters defined above and the melting point $\theta_{m}$ for selected materials. ${ }^{10,11}$

Heat conduction during compaction has been neglected in our analyses. This approximation is valid for large powder particles on the order of tens of microns, where the thermal diffusion distance, $\chi$ is small compared with the characteristic length of the microstructure which is the average pore size. The value of $\chi$ is given by $\sqrt{\kappa t_{s}}$, where $\kappa$ is the thermal diffusivity and $t_{s}$ is the shock rise time. The thermal diffusivity $\kappa$ is related to the thermal conductivity $K$ by $\kappa=K /\left(\rho_{0} c_{p}\right)$, where $\rho_{0}$ and $c_{p}$ are the solid density and heat capacity, respectively. Table II summarizes these thermal properties and the shock rise times for the materials listed previously in Table I. In our present analysis, it is assumed that the dynamic pore collapse occurs adiabatically. ${ }^{12}$

Figure 1 compares shock rise times calculated using our analysis ${ }^{12}$ with the experimental data ${ }^{8}$ on $2 \mathrm{Al}+\mathrm{Fe}_{2} \mathrm{O}_{3}$ powder mixtures. The shock pressure rate $\dot{p}$ is calculated by dividing the shock pressure with the measured shock rise time. Due to the asymptotic nature of pore collapse process, the calculated shock rise time $t_{s}$ is determined to be the time when the final relative density $\bar{\rho}_{s} \geqslant 98 \%$. Also shown in Fig. 1 are calculated shock rise times resulting from quasistatic analyses of pore collapse. ${ }^{7,13}$ The quasistatic analysis of pore collapse in rate-independent, perfectly plastic materials gives the shock rise time as ${ }^{7}$

$$
t_{s}=\frac{2}{\sqrt{3}} \frac{\tau_{0}}{\dot{p}} \ln \left(\frac{1}{1-\bar{\rho}_{s}}\right) .
$$

Wilkinson and Ashby ${ }^{13}$ conducted the quasistatic analysis of pore collapse for rate-dependent, perfectly plastic materials and the corresponding shock rise time can be determined by solving the following equations: ${ }^{12,13}$

$$
\begin{aligned}
& \dot{\bar{\rho}}=\frac{\sqrt{3}}{2} \dot{\gamma}_{0} \frac{\bar{\rho}(1-\bar{\rho})}{\left[1-(1-\bar{\rho})^{m}\right]^{1 / m}}\left(\frac{\sqrt{3} m}{2} \frac{\dot{p} t}{\tau_{0}}\right)^{1 / m}, \\
& \bar{\rho}\left(t_{s}\right)=\bar{\rho}_{s}, \quad 0<m \leqslant 1 .
\end{aligned}
$$

TABLE I. Viscoplastic parameters for matrix materials (Refs. 10 and 11).

\begin{tabular}{lccccccc}
\hline \hline Material & $\begin{array}{c}\tau_{0} \\
(\mathrm{MPa})\end{array}$ & $\begin{array}{c}\dot{\gamma}_{0} \\
\left(10^{5} \mathrm{~s}^{-1}\right)\end{array}$ & $m$ & $\gamma_{0}$ & $n$ & $\nu$ & $\begin{array}{c}\theta_{m} \\
(\mathrm{~K})\end{array}$ \\
\hline Aluminum & 125 & 1.53 & 0.254 & 0.05 & 0.04 & -0.4 & 933 \\
Copper & 270 & 5.0 & 0.20 & 0.10 & 0.10 & -0.5 & 1358 \\
Iron & 420 & 4.0 & 0.20 & 0.15 & 0.085 & -0.6 & 1809 \\
\hline \hline
\end{tabular}


TABLE II. Selected thermal properties and pore-collapse parameters of matrix materials.

\begin{tabular}{lcccccc}
\hline \hline Material & $\begin{array}{c}\rho_{0} \\
\left(\mathrm{~kg} / \mathrm{m}^{3}\right)\end{array}$ & $\begin{array}{c}c_{p} \\
(\mathrm{~J} / \mathrm{kg} / \mathrm{K})^{\mathrm{a}}\end{array}$ & $\begin{array}{c}K \\
(\mathrm{~W} / \mathrm{m} / \mathrm{K})^{\mathrm{a}}\end{array}$ & $\begin{array}{c}\kappa, 10^{-4} \\
\left(\mathrm{~m}^{2} / \mathrm{s}\right)^{\mathrm{a}}\end{array}$ & $\begin{array}{c}t_{s} \\
(\mathrm{~ns})^{\mathrm{b}}\end{array}$ & $\begin{array}{c}\chi \\
(\mu \mathrm{m})\end{array}$ \\
\hline Aluminum & 2700 & $893-1076$ & 238 & $0.96-0.82$ & 31 & $1.7-1.6$ \\
Copper & 8940 & $383-473$ & $394-244$ & $1.15-0.58$ & 36 & $2.0-1.4$ \\
Iron & 7800 & $444-791$ & $73.3-30$ & $0.22-0.05$ & 54 & $1.1-0.6$ \\
\hline
\end{tabular}

${ }^{a}$ Heat capacity, thermal conductivity, and thermal diffusivity are listed for both room temperature and elevated temperature $\left(\theta / \theta_{m} \approx 0.7-0.8\right)$.

${ }^{\mathrm{b}}$ The shock rise time $t_{s}$ is estimated from Eq. (3) for $\dot{p}=75 \mathrm{MPa} / \mathrm{ns}$.

Inspection of Fig. 1 indicates that our analysis ${ }^{12}$ gives a better estimate of shock rise times over a wide range of loading rates by including both dynamic effects and the rate dependence of flow strength of the matrix material. For loading rates less than $20 \mathrm{MPa} / \mathrm{ns}$, the effect of rate becomes important; at higher loading rates, dynamic effects begin to dominate. The high loading rate $\dot{p}_{0}$ above which the shock rise time tends to be nearly constant, say $d t_{s} / d \dot{p} \leqslant 1 \mathrm{~ns} / \mathrm{MPa} / \mathrm{ns}$, can be estimated for a porous material using either Eq. (2):

$$
\dot{p}_{0}=\sqrt{\frac{2}{\sqrt{3}} \tau_{0} \ln \left(\frac{1}{1-\bar{\rho}_{s}}\right)},
$$

or Eq. (3):

$$
\begin{aligned}
\dot{p}_{0}= & \left(\frac{1}{1+m}\right)^{(1+m) /(2+m)} \alpha^{m /(1+m)}, \\
\alpha= & {\left[\left(1+\frac{1}{m}\right)\left(\frac{2 \tau_{0}}{\sqrt{3} m}\right) \frac{2}{\sqrt{3} \dot{\gamma}_{0}}\right] } \\
& \times \int_{\bar{\rho}_{0}}^{\overline{\rho_{s}}} \frac{\left(1-(1-\bar{\rho})^{m}\right]^{1 / m}}{\bar{\rho}(1-\bar{\rho})} d \bar{\rho} .
\end{aligned}
$$

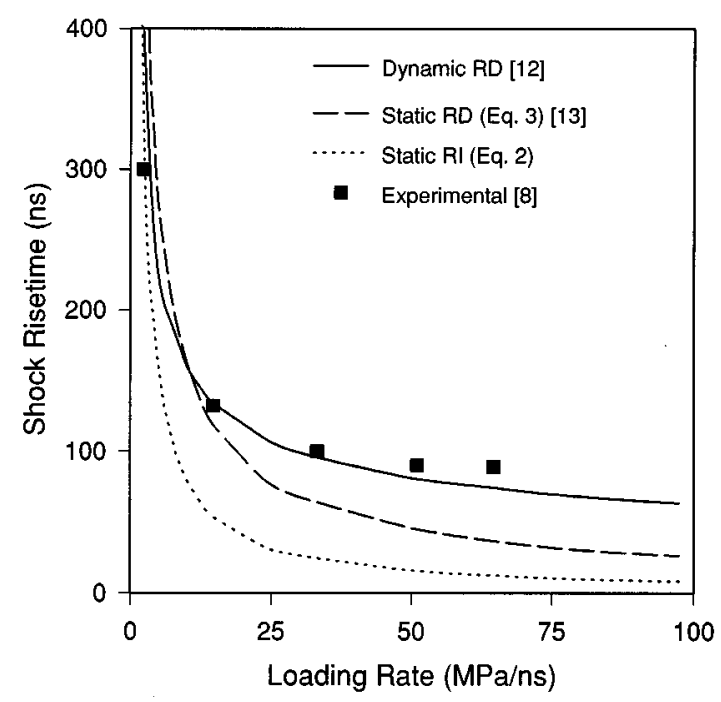

FIG. 1. The shock rise time of a porous aluminum-based material over a wide range of dynamic loading rates. The powder was initially at the ambient temperature, its porosity is $47 \%$ and a higher reference shear stress $\left[\tau_{0}=175 \mathrm{Mpa}\right.$ in Eq. (1)] was assumed to account for the $2 \mathrm{Al}+\mathrm{Fe}_{2} \mathrm{O}_{3}$ powder mixture reported in the experiment (Ref. 8).

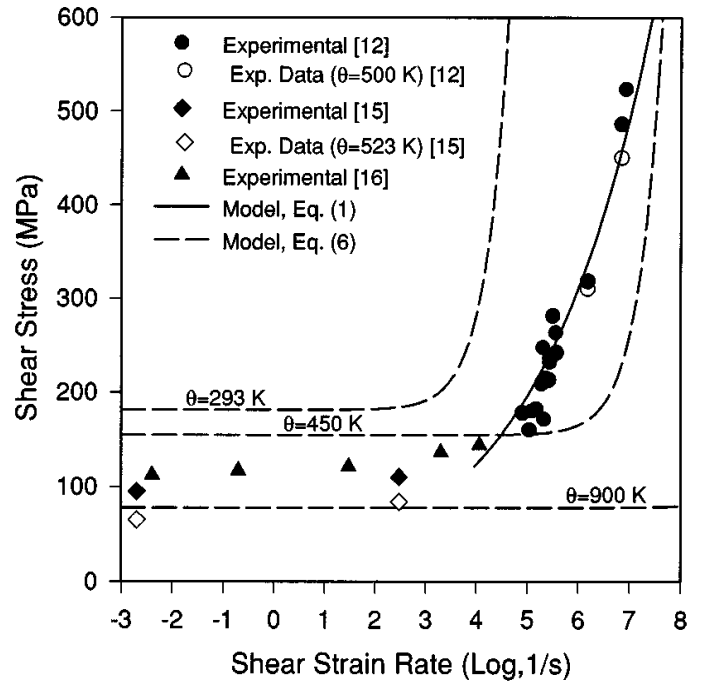

FIG. 2. A summary of rate sensitivity of copper over a wide range of strain rates. All data are for room temperature except where noted otherwise.

Carroll, Kim, and Nesterenko ${ }^{14}$ extended the dynamic pore collapse analysis of Carroll and Holt ${ }^{9}$ to an incompressible, perfectly viscoplastic material with temperaturedependent yield strength and viscosity. Using the present notation, their model can be expressed as

$$
\tau=\tau_{i}\left(1-\frac{\theta}{\theta_{m}}\right)+\eta_{m} \dot{\gamma} \exp \left[-\frac{1}{B}\left(\frac{1}{\theta}-\frac{1}{\theta_{m}}\right)\right],
$$

where $\tau_{i}, \eta_{m}$, and $B$ are material constants. They assumed a strong temperature dependence of the viscosity and the strength. ${ }^{14}$ Their calculations indicated a significant difference in the compaction process depending on whether viscoplastic or inertial effects are dominant for materials initially at room temperature and elevated temperature.

The above conclusion is largely due to the assumed strong thermal softening effects of copper at very high strain rates. However, there is very limited data on the flow strength of copper at both high strain rates $\left(10^{5}-10^{7} \mathrm{~s}^{-1}\right)$ and elevated temperatures. Existing experimental evidence indicates that the flow strength remains relatively high and strongly rate dependent under these conditions. ${ }^{10,11,15,16}$ Figure 2 summarizes the variation of flow stress with shear strain rate for copper over a wide range of strain rates. The prediction of Eq. (6) for both room temperature $(293 \mathrm{~K})$ and elevated temperatures $(450$ and $900 \mathrm{~K}){ }^{14}$ as well as the model prediction of Eq. (1) is shown in Fig. 2. Clearly, the prediction of Eq. (6) overestimates the flow strength of copper at room temperature by assuming a linear dependence of flow stress on strain rate while greatly underestimating the flow strength of copper at 450 and $900 \mathrm{~K}$ by assuming a very strong thermal softening effect.

The dynamic compaction of copper powders ${ }^{14}$ was reanalyzed using the material model given by Eq. (1) and the formulation and solution procedures given by Tong and Ravichandran. ${ }^{12}$ The estimated shock rise times are shown in Fig. 3. By using the more realistic viscoplastic material model, the magnitude of the calculated shock rise times and 


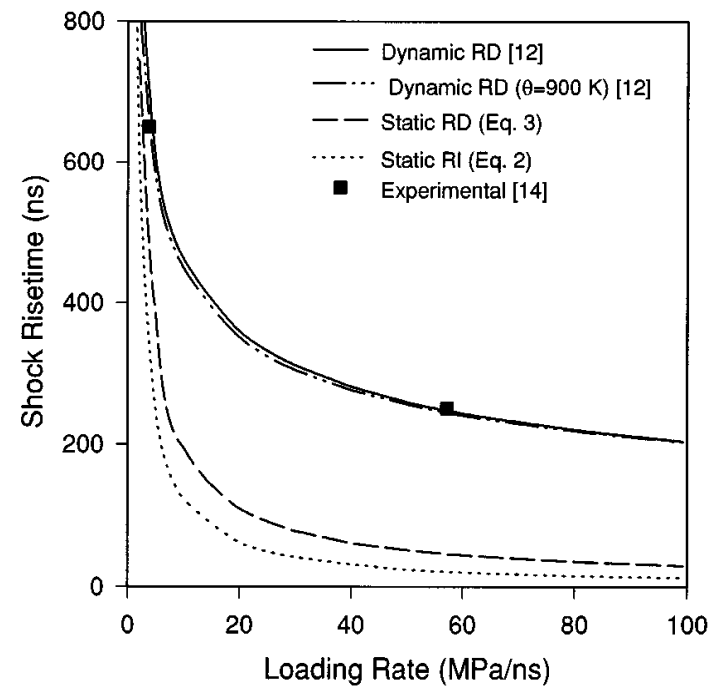

FIG. 3. The shock rise time of copper powders over a wide range of dynamic loading rates. The initial powder porosity is $50 \%$ and an average pore size of $0.35 \mathrm{~mm}$ is used in all dynamic analyses for the copper powders with sizes ranging from 0.1 to $1 \mathrm{~mm}$ (Ref. 14). All data are for room temperatures except where noted otherwise.

their dependence on loading rate are found to be consistent with the experimental data. Similar agreement was obtained when the initial powder temperature was assumed to be either 450 or $900 \mathrm{~K}$. It is suggested that any reduction of the shock rise time due to thermal softening is counteracted by the increasing retardation effects of inertia, especially at high loading rates. Inertial effects are found to be dominant in the compaction of both room-temperature and preheated copper powders.

In summary, the recently developed formulation of finite elastic/viscoplastic deformation of a spherical shell and related numerical solution procedures ${ }^{12}$ are useful in examin- ing the effects of dynamic loading rate and initial powder temperature on the shock rise time in porous materials. The shock rise time is found to decrease with increasing shock pressure and become relatively constant at high shock pressures. The dependence of the shock rise time on the initial temperature of porous materials is found to be weak. Results of our analysis show good agreement with the time-resolved shock rise time measurements. ${ }^{8,14}$

The authors would like to acknowledge the support for this research by the Division of Materials Research of the National Science Foundation through Grant No. DMR9396132, Program Director, Dr. B. MacDonald.

${ }^{1}$ V. D. Linse, Dynamic Compaction of Metal and Ceramic Powders, NMAB-394 (National Academy, Washington, DC, 1983).

${ }^{2}$ W. H. Gourdin, Prog. Mater. Sci. 30, 39 (1986).

${ }^{3}$ N. N. Thadhani, Prog. Mater. Sci. 37, 117 (1993).

${ }^{4}$ W. Tong, G. Ravichandran, T. Christman, and T. Vreeland, Jr., Acta Metall. Mater. (to be published).

${ }^{5}$ W. Herrmann, J. Appl. Phys. 40, 2490 (1969).

${ }^{6}$ R. G. McQueen, S. P. Marsh, and W. J. Carter, Symposium on High Dynamic Pressure, IUTAM, Paris, France, September, 1967 (Gordon and Breach, New York, 1968), p. 67.

${ }^{7}$ B. M. Butcher and C. H. Karnes, J. Appl. Phys. 40, 2967 (1969).

${ }^{8}$ G. T. Holman, Jr., R. A. Graham, and M. U. Anderson, Proceedings of the Joint Meeting of the International Association for the Advancement of High Pressure Science and Technology and the American Physical Society Topical Group on Shock Compression of Condensed Matter, edited by S. C. Schmidt et al., Denver, CO, 1993 (AIP Press, New York, 1994).

${ }^{9}$ M. M. Carroll and A. C. Holt, J. Appl. Phys. 43, 1626 (1972).

${ }^{10}$ R. W. Klopp, R. J. Clifton, and T. G. Shawki, Mech. Mater. 4, 375 (1985).

${ }^{11}$ W. Tong, R. J. Clifton, and S. Huang, J. Mech. Phys. Solids 40, 1251 (1992).

${ }^{12}$ W. Tong and G. Ravichandran, J. Appl. Phys. 74, 2425 (1993).

${ }^{13}$ D. S. Wilkinson and M. F. Ashby, Acta Metall. 23, 1277 (1975).

${ }^{14}$ M. M. Carroll, K. T. Kim, and V. F. Nesterenko, J. Appl. Phys. 59, 1962 (1986).

${ }^{15}$ P. E. Senseny, J. Duffy, and R. H. Hawley, ASME J. Appl. Mech. 45, 60 (1978).

${ }^{16}$ P. S. Follansbee and U. F. Kocks, Acta Metall. 36, 81 (1988). 